\title{
Como anda o cuidado prestado pelos médicos aos pacientes com demência na
}

\section{Atenção Primária?}

\author{
How is the care provided by physicians to patients with dementia in Primary Care? \\ ¿Cómo proporcionan los médicos la atención a los pacientes con demencia en Atención Primaria?
}

Recebido: 14/12/2021 | Revisado: 19/12/2021 | Aceito: 27/12/2021 | Publicado: 05/01/2022

\author{
Claudia Cristina Ferreira Ramos \\ ORCID: https://orcid.org/0000-0003-3183-6970 \\ Universidade Municipal de São Caetano do Sul, Brasil \\ E-mail: claudia.ramos@online.uscs.edu.br \\ Rosamaria Rodrigues Garcia \\ ORCID: https://orcid.org/0000-0001-9454-6810 \\ Universidade Municipal de São Caetano do Sul, Brasil \\ E-mail: rosamaria.garcia@online.uscs.edu.br
}

\begin{abstract}
Resumo
Introdução: a incidência de doenças neurogenerativas vêm sofrendo aumento progressivo em decorrência do envelhecimento populacional. Deste modo, é imprescindível o estabelecimento de protocolos de rastreio cognitivo para identificação de alterações cognitivas e comportamentais, diagnóstico precoce e tratamento imediato, no acompanhamento da pessoa idosa, principalmente nos serviços de Atenção Primária à Saúde. Objetivos: Descrever o atitudes e perfil profissional de médicos no cuidado aos pacientes com demência, no município de São Caetano do Sul, na atenção primária. Método: estudo quantitativo, transversal, descritivo, com amostra não probabilística, coletada por conveniência, por meio da aplicação do instrumento "Atenció Sanitària de Les Demències: la visió de L' Atenció Primarià - para médicos - versão brasileira", aos médicos da Atenção Primária de São Caetano do Sul. Resultados: houve $100 \%$ de participação da Atenção Primária, totalizando 28 médicos, sendo $50 \%$ do genêro feminino. Os participantes possuem 82 meses, em média, de atuação na Atenção Primária. Observou-se que 82,6\% referiram que fazem com maior frequência diagnóstico de ususários com demência em quadro moderado; $68,2 \%$ relataram dificuldades para acompanhar clinicamente usuários com quadro demencial grave e $87 \%$ mencionaram que referenciam usuários para médicos neurologistas para realização do diagnóstico. Parte dos médicos $(39,1 \%)$ relatou indicam mais de $75 \%$ dos pacientes com demência para serviços de referência. Ainda que a totalidade da amostra compreendam a importância e a necessidade, $34,8 \%$ ainda não cursaram atualização sobre a temática da demência. As principais dificuldades relatadas pelos médicos estão relacionadas à prescrição medicamentosa, à necessidade de visitas frequentes, e à complexidade do cuidado. Conclusão: Observou-se lacunas na identificação de quadros demenciais, bem como no acompanhamento clínico adequado aos pacientes, gerando direcionamentos desqualificados para a atenção secundária, reduzindo a resolutividade da atenção primária para tal demanda. Ficou evidente a necessidade preemente de atualização dos médicos, visnado melhoria da qualidade do cuidado prestado.

Palavras-chave: Atenção à saúde; Estratégia saúde da família; Demência; Prática integral de cuidados de saúde; Garantia da qualidade dos cuidados de saúde.
\end{abstract}

\begin{abstract}
Introduction: the incidence of neurogenerative diseases has been progressively increasing as a result of population aging. Thus, it is essential to establish cognitive screening protocols to identify cognitive and behavioral changes, early diagnosis and immediate treatment, in monitoring the elderly, especially in Primary Health Care services. Objectives: To describe the attitudes and professional profile of doctors in the care of patients with dementia, in the city of São Caetano do Sul, in primary care. Method: quantitative, cross-sectional, descriptive study, with a nonprobabilistic sample, collected for convenience, through the application of the instrument "Atenció Sanitària de Les Demències: la visió de L'Atenció Primarià - for physicians - Brazilian version", to healthcare physicians Primary Care in São Caetano do Sul. Results: there was 100\% participation in Primary Care, totaling 28 doctors, $50 \%$ of which were female. Participants have, on average, 82 months of experience in Primary Care. It was observed that $82.6 \%$ reported that they more frequently diagnose users with dementia in a moderate condition; $68.2 \%$ reported difficulties in clinically monitoring users with severe dementia and $87 \%$ mentioned that they refer users to neurologists for diagnosis. Part of physicians (39.1\%) reported referring more than $75 \%$ of patients with dementia to referral services. Even though the entire sample understands the importance and need, $34.8 \%$ have not yet taken an update on the topic of dementia. The main difficulties reported by physicians are related to drug prescription, the need for frequent visits,
\end{abstract}


and the complexity of care. Conclusion: There were gaps in the identification of dementia, as well as in the adequate clinical monitoring of patients, generating unqualified directions for secondary care, reducing the resolution of primary care for such a demand. The urgent need to update physicians was evident, with a view to improving the quality of care provided.

Keywords: Delivery of health care; Family health strategy; Dementia; Integral healthcare practice; Quality assurance health care.

\section{Resumen}

Introducción: la incidencia de enfermedades neurogenerativas ha ido aumentando progresivamente como consecuencia del envejecimiento de la población. Por ello, es fundamental establecer protocolos de cribado cognitivo para identificar cambios cognitivos y conductuales, diagnóstico precoz y tratamiento inmediato, en el seguimiento de las personas mayores, especialmente en los servicios de Atención Primaria de Salud. Objetivos: Describir las actitudes y perfil profesional de los médicos en la atención de pacientes con demencia, en la ciudad de São Caetano do Sul, en atención primaria. Método: estudio cuantitativo, transversal, descriptivo, con muestra no probabilística, recogida por conveniencia, mediante la aplicación del instrumento "Atenció Sanitària de Les Demències: la visió de L'Atenció Primarià - para médicos - versión brasileña", a médicos de atención primaria de la salud en São Caetano do Sul. Resultados: hubo 100\% de participación en Atención Primaria, totalizando 28 médicos, 50\% de los cuales eran mujeres. Los participantes tienen, en promedio, 82 meses de experiencia en Atención Primaria. Se observó que el $82,6 \%$ refirió que diagnostican con mayor frecuencia a los usuarios con demencia en estado moderado; El 68,2\% reportó dificultades para monitorear clínicamente a los usuarios con demencia severa y el $87 \%$ mencionó que derivan a los usuarios a neurólogos para su diagnóstico. Una parte de los médicos $(39,1 \%)$ informó haber remitido a más del $75 \%$ de los pacientes con demencia a los servicios de derivación. Si bien toda la muestra comprende la importancia y la necesidad, el 34,8\% aún no ha actualizado el tema de la demencia. Las principales dificultades reportadas por los médicos están relacionadas con la prescripción de medicamentos, la necesidad de visitas frecuentes y la complejidad de la atención. Conclusión: Existieron vacíos en la identificación de las demencias, así como en el adecuado seguimiento clínico de los pacientes, generando direcciones no calificadas para la atención secundaria, reduciendo la resolución de la atención primaria para tal demanda. Era evidente la urgente necesidad de actualizar a los médicos, con miras a mejorar la calidad de la atención brindada.

Palabras clave: Atención a la salud; Estrategia de salud familiar; Demencia; Práctica integral de atención; Garantía de la calidad de atención de salud.

\section{Introdução}

O processo do envelhecimento humano é caracterizado por alterações do sistema nervoso central que modificam aspectos sensoriais e motores, emocionais e cognitivos, como no caso da demência. Com o passar dos anos, a cognição sofre mudanças significativas que afetam as atividades diárias dos pacientes e familiares. Sendo assim, é fundamental a avaliação adequada das funções cognitivas e comportamentais no seguimento de indivíduos idosos para mensurar possíveis déficits cognitivos, realizar diagnóstico precoce e indicar tratamento adequado e acompanhamento multiprofissional do paciente quando necessário (Unwin, Loskutova, Knicely \& Wood, 2019).

Devido à acelerada transição demográfica, as doenças decorrentes do processo de envelhecimento se tornam um ponto de fundamental importância levando a uma forte demanda sobre serviços primários de saúde (Brucki, 2013).

No caso da demência, o envelhecimento provavelmente é o fator de maior risco e já se encontra entre os transtornos neuropsiquiátricos mais comuns em idosos (Livingston et al., 2020).

Dessa forma, é de suma importância que o médico da atenção primária esteja preparado para avaliar e conduzir os pacientes com diagnóstico de Síndrome Demencial, assim como realizar orientações nas situações em que for detectado alguma dificuldade cognitiva leve sem prejuízo da funcionalidade (Suemoto et al., 2017).

A análise conjunta da anamnese clínica, exames complementares e resultado da aplicação de escalas de avaliação cognitiva auxiliam para diagnóstico correto do paciente assim como em sua assistência adequada (US Preventive Services Task Force et al., 2020).

A implementação da capacitação dos médicos na atenção primária de saúde do município favorece a reorganização de protocolos dos serviços, pois por meio do rastreamento multidimensional dos pacientes é possível a integração dos 
profissionais de saúde ampliando o processo colaborativo entre eles (US Preventive Services Task Force et al., 2020).

A avaliação do conhecimento médico nos cuidados primários em relação às demências, pode ser realizada através da aplicação do questionário "Atenció Sanitària de Les Demències: la visió de L' Atenció Primarià" que aborda a temática de forma bem completa, além de disponibilizar duas versões para a avaliação: uma destinada a médicos e outra, a enfermeiros (Tuero et al., 2011).

Essa escala foi elaborada pela Sociedade Catalã de Medicina Familiar e Comunitária de maneira a conhecer as necessidades dos profissionais no atendimento desse perfil de pacientes, sendo validada para o português em 2014. Apresenta questões abertas e fechadas relacionadas ao acompanhamento de pacientes com demência e de seus cuidadores. Não existem respostas incorretas, pois a escala é baseada na opinião dos profissionais em relação a sua prática clínica (Costa et al., 2015).

O objetivo do presente estudo é descrever as dificuldades no cuidado de pacientes com demência, apontadas pelos médicos que atuam na atenção primária do Sistema Único de Saúde do município de São Caetano do Sul, através do instrumento Atenció Sanitària de Les Demències: la visió de L' Atenció Primarià - versão brasileira.

\section{Metodologia}

Trata-se de estudo transversal, observacional e descritivo, de abordagem quantitativa, com amostra não aleatória, coletada por conveniência, realizado a partir dos preceitos e etapas do método científico, em que se busca respostas a problemas percebidos na sociedade, delimitados pelo pesquisador e que requerem investigação, sendo submetidos às etapas do método científico (Koche, 2011).

Para a realização da pesquisa científica são previstas etapas dispostas de forma sistematizada, racional e estabelecidas a partir da lógica. Dentre elas, estão a identificação do tema ou a problematização do objeto de estudo, o levantamento bibliográfico sobre o que já existe na literatura a respeito, o desenho de pesquisa, a escolha e o desenvolvimento do método científico, a coleta, tabulação, análise e interpretação dos dados, a discussão dos resultados a partir da fundamentação teórica e da literatura existente, as considerações finais e a divulgação da pesquisa (Freire e Pattussi, 2018).

O estudo foi realizado com os 28 médicos da Atenção Primária do Sistema Único de Saúde do município de São Caetano do Sul que atuam nas equipes da Estratégia de Saúde da Família das 10 Unidades Básicas de Saúde (Amélia R. Locatelli, Dr. Angelo Antenor Zambom, Caterina Dall Anese, Darcy Sarmanho Vargas, Dolores Massei, João Luiz Pasqual Bonaparte, Maria Corbeta Segato, Moacir Gallina, Nair Spina Benedicts e Dr. Ivanhoé Esposito), Centro de Especialidades Médicas e Centro Policlínico Gentil Rstom.

Foram coletados dados demográficos como idade, sexo e todos serão convidados a responderem ao questionário "Instrumento Atenció Sanitària de Les Demències: la visió de L' Atenció Primarià - para médicos - versão brasileira", presencialmente com duração de preenchimento de aproximadamente 25 minutos. Os participantes responderam as perguntas após a leitura e assinatura do termo de consentimento livre e esclarecido.

O questionário do presente estudo coletou informações sobre tempo de formação médica e atuação profissional, conhecimento sobre demências, características dos pacientes idosos e de pacientes com demência atendidos, quais as maiores dificuldades dos participantes na realização do diagnóstico, tratamento e manejo dos pacientes com demência na Atenção Básica, quais os testes cognitivos, de capacidade funcional e exames complementares mais utilizados pelos participantes para fazer rastreio e acompanhamento dos pacientes. O questionário também investiga as formas de encaminhamento para atendimento especializado mais utilizadas pelos participantes, bem como as características do manejo de pacientes com demência na atenção especializada. Por fim, o questionário apresenta um caso clínico para que o participante indique sua conduta e intervenções. 
$\mathrm{O}$ participante teve assegurado o direito de não responder à pergunta e garantido o direito de acesso ao teor do conteúdo do instrumento (tópicos que foram abordados) antes de responder às perguntas, para uma tomada de decisão informada.

A pesquisa envolveu risco mínimo de possível desconforto ao responder alguma pergunta do questionário proposto, ou do tempo disponibilizado para preenchimento do instrumento. O participante teve a liberdade para não responder; interromper a pesquisa; fazer pausas; ou cancelar a sua participação a qualquer momento. Em todos esses casos, o participante não foi prejudicado, penalizado ou responsabilizado de nenhuma forma. Como benefício, será realizada uma capacitação com aulas teóricas e práticas sobre o cuidado das demências na Atenção Primária, a ser ofertada de forma on-line aos participantes de pesquisa.

Foram critérios de inclusão dos participantes: médicos de ambos os gêneros, que atuassem na Atenção Primária do Sistema Único de Saúde do município de São Caetano do Sul, independente do tempo de atuação no serviço; médicos da Atenção Primária de São Caetano do Sul que leram e assinaram eletronicamente o Termo de Consentimento Livre e Esclarecido, aceitando participar do estudo, após receberem e aceitarem ao convite para participarem da pesquisa.

A pesquisa envolveu risco mínimo de possível desconforto ao responder alguma pergunta do questionário proposto, ou do tempo disponibilizado para preenchimento do instrumento. Caso isso tenha ocorrido, o participante teve a liberdade para não responder; interromper a pesquisa; fazer pausas; ou cancelar a sua participação a qualquer momento. Em todos esses casos, o participante não foi prejudicado, penalizado ou responsabilizado de nenhuma forma.

A análise dos dados foi realizada utilizando-se métodos de estatística descritiva de variáveis categóricas.

O entendimento da atuação médica relacionada aos pacientes com diagnóstico de demência da atenção primária possibilita a implementação de capacitação direcionada sobre essa temática, o que favorece o estabelecimento de fluxo dos protocolos dos serviços e assim melhor assistência para esses pacientes e seus cuidadores.

\section{Resultados}

Foram coletados dados de 28 médicos da Atenção Básica do município de São Caetano do Sul, totalizando 100\% da equipe médica que compõe a Estratégia de Saúde da Família (ESF).

Os dados sociodemográficos tiveram como objetivo caracterizar a população que, voluntariamente, aceitou participar da pesquisa. Observou-se que 50\% participantes era do sexo feminino.

Todos os médicos avaliados realizaram a graduação em Medicina no Brasil, a maioria (65\%) tem idade entre 25 e 35 anos. Todos os participantes relataram achar necessário realizar atividades de capacitação específica em demência, embora 46,4\% referem ter realizado essa atividade há mais de 2 anos, 28,6\% referem nunca ter participado de capacitação, 17,9\% participaram da capacitação no último ano e 7,1\% referem ter realizado essa atividade há mais de 5 anos.

Já no que diz respeito ao momento mais comum para o diagnóstico dos quadros demenciais: 78,6\% dos participantes apontaram que é mais frequente realizarem o diagnóstico em fase moderada da evolução da doença, 14,3\% relataram diagnosticar na fase leve e 7,1\% referiram não realizar o diagnóstico de demência tendo preferência em encaminhar esses pacientes ao serviço especializado.

Também foi possível avaliar através do questionário os instrumentos de avaliação cognitiva mais utilizados na prática clínica dos médicos avaliados e pode-se notar que 100\% dos indivíduos utilizam o Miniexame do Estado Mental, 64,3\% aplicam o Teste do desenho do relógio, 46,4\% fazem a Avaliação cognitiva funcional global, 21,4\% realizam o Teste de fluência verbal categoria animal e/ou frutas, 14,3\% aplicam o MOCA, 7,1\% utilizam o Teste de nomeação de Boston e o Questionário de avaliação do comprometimento cognitivo do idoso por informante (IQCODE) e 3,6\% realizam a Lista de 
palavras do CERAD e Escala Isquêmica de Hachinski. Todos os médicos relataram utilizar alguma escala para avaliação da função cognitiva.

Também foi possível avaliar os participantes em relação a capacidade funcional, 57,1\% dos médicos referiram habitualmente não utilizar nenhum teste para avaliação, 25\% utilizam o Índice de Katz, 17,9\% aplicam o Teste de Lawton, 10,7\% realizam o Teste de Pfeffer e nenhum profissional apontou o uso do Índice de Barthel, como mostra o Gráfico 1.

Gráfico 1: Distribuição dos médicos da Atenção Primária do município de São Caetano do Sul de acordo com a frequência de testes habitualmente utilizados na prática clínica, para avaliar a capacidade funcional de pacientes. São Caetano do Sul. 2021.

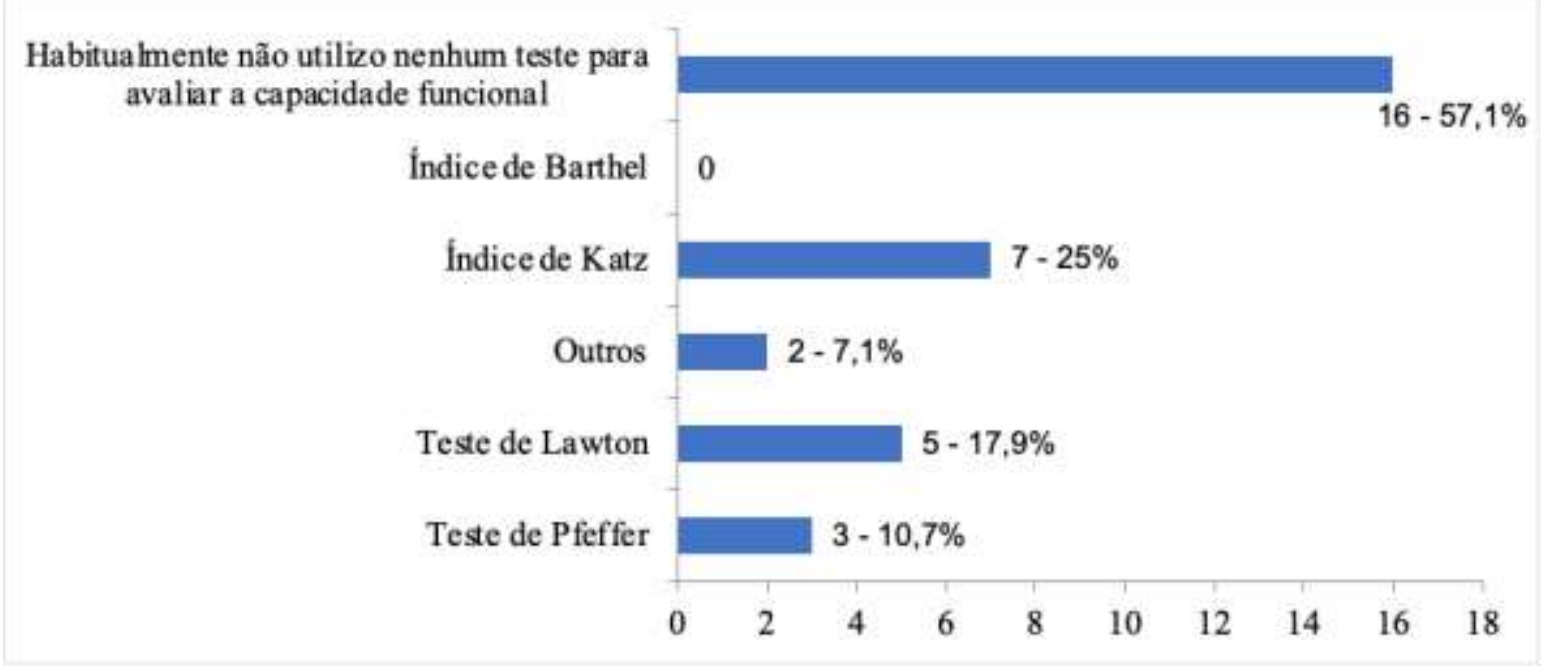

Fonte: Dados de pesquisa.

Observou-se que 70,4\% dos profissionais encontram dificuldades para cuidar de pacientes com demência grave sendo o principal motivo apontado por $68,4 \%$ dos médicos foi a complexidade do tratamento farmacológico exigido nesses casos. Também foi visto que 36,8\% referiram que a demanda de pacientes graves não são solucionáveis na Atenção Básica, 26,3\% relatam que não há suporte de atendimento especializado e 15,8\% apontaram como dificuldade a exigência de múltiplas visitas domiciliares, de acordo com Gráfico 2. 
Gráfico 2: Distribuição dos médicos da Atenção Primária do município de São Caetano do Sul, de acordo com os motivos atribuídos às dificuldades de cuidado de pacientes com demência. São Caetano do Sul. 2021.

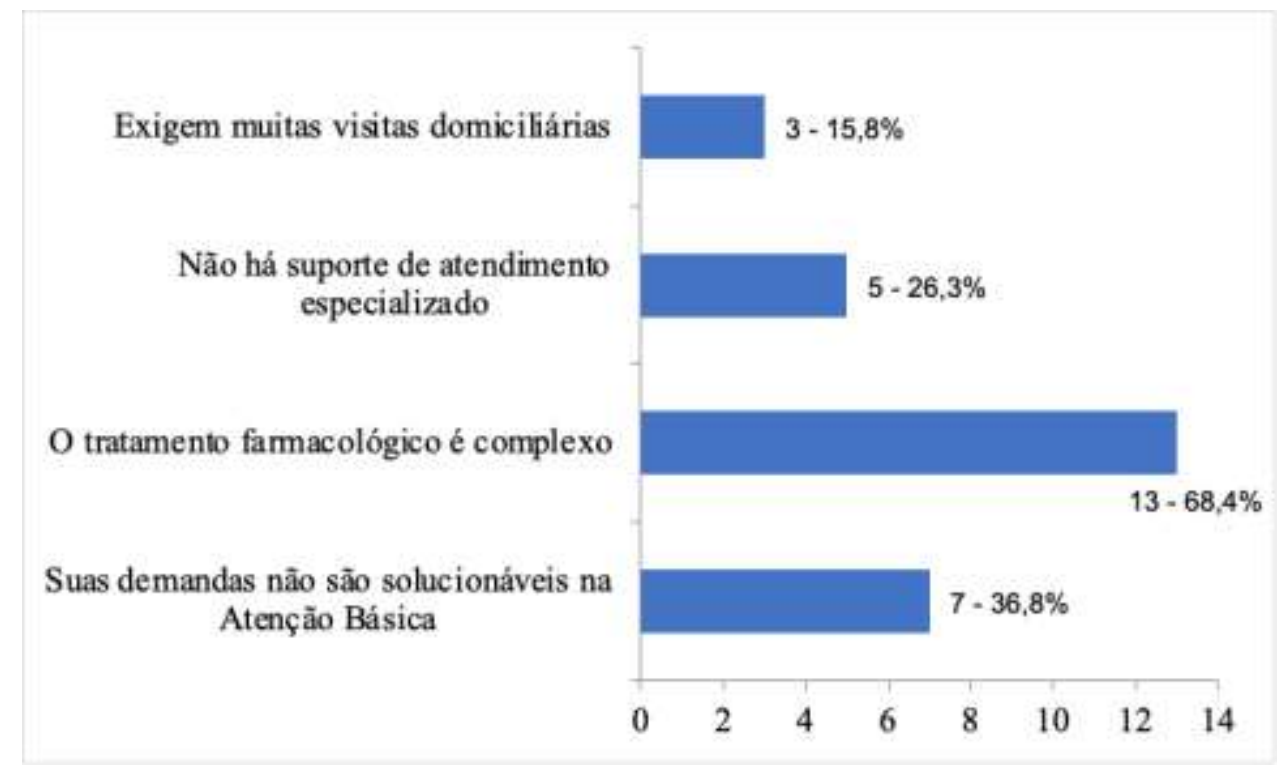

Fonte: Dados de pesquisa.

O Gráfico 3 apresenta dados em relação ao encaminhamento dos pacientes com quadro de comprometimento cognitivo realizado pelos médicos da Atenção Básica e foi visto que 89,3\% encaminham para o neurologista de referência, $50 \%$ dos médicos indicam para o Centro de Referência do Idoso, 7,1\% para o Ambulatório Médico de Especialidades e Ambulatório de Especialidades em Demências e 3,6\% encaminham para o Serviço de Neurologia Hospitalar.

Gráfico 3: Distribuição da frequência de serviços médicos especializados na área de referência para encaminhamento de pacientes atendidos por médicos da Atenção Primária do município de São Caetano do Sul visando o diagnóstico e/ou controle de demência. São Caetano do Sul. 2021.

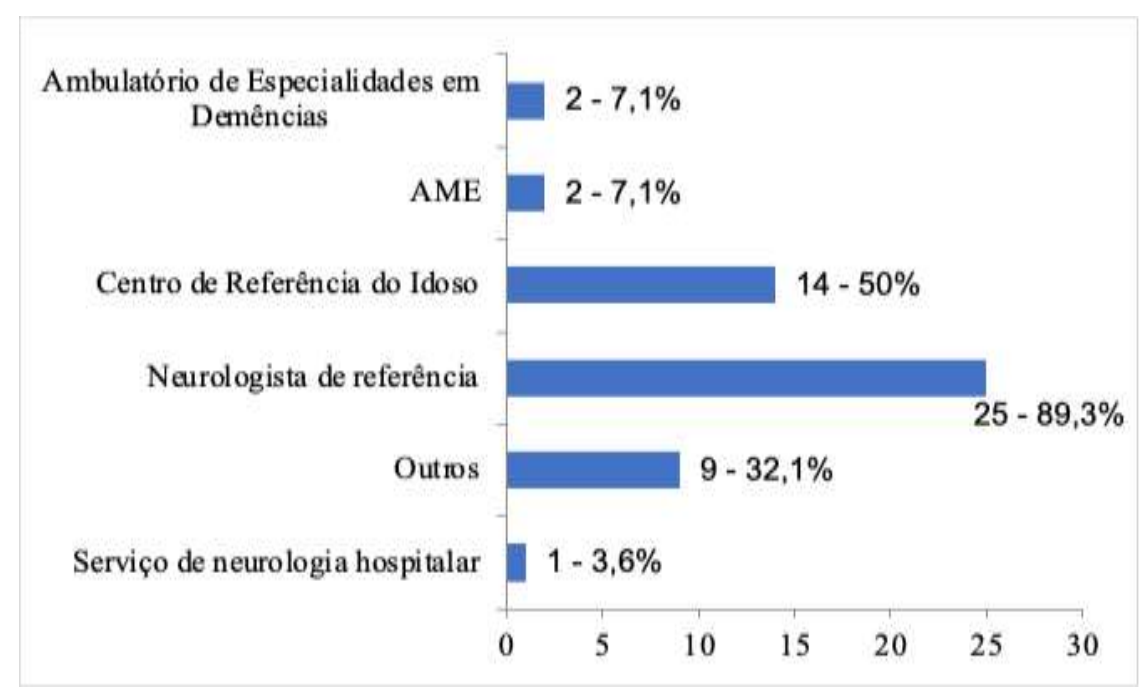

Fonte: Dados de pesquisa.

Já em relação a porcentagem de paciente com demência que são encaminhados aos serviços especializados, foi apontado que 32,1\% dos profissionais encaminham mais de 75\% dos pacientes para confirmação do diagnóstico, 21,4\% dos 
médicos referiram encaminhar menos de $10 \%$ dos casos, 17,9\% relatam de 11 a $25 \%$, 10,7\% de 51 a $75 \%$ e $7,1 \%$ responderam que não encaminham nenhum caso de demência aos serviços especializados, de acordo com o gráfico 4.

Gráfico 4: Distribuição dos médicos da Atenção Primária do município de São Caetano do Sul, de acordo com o percentual de pacientes com demência encaminhados aos serviços especializados para confirmação do diagnóstico. São Caetano do Sul. 2021.

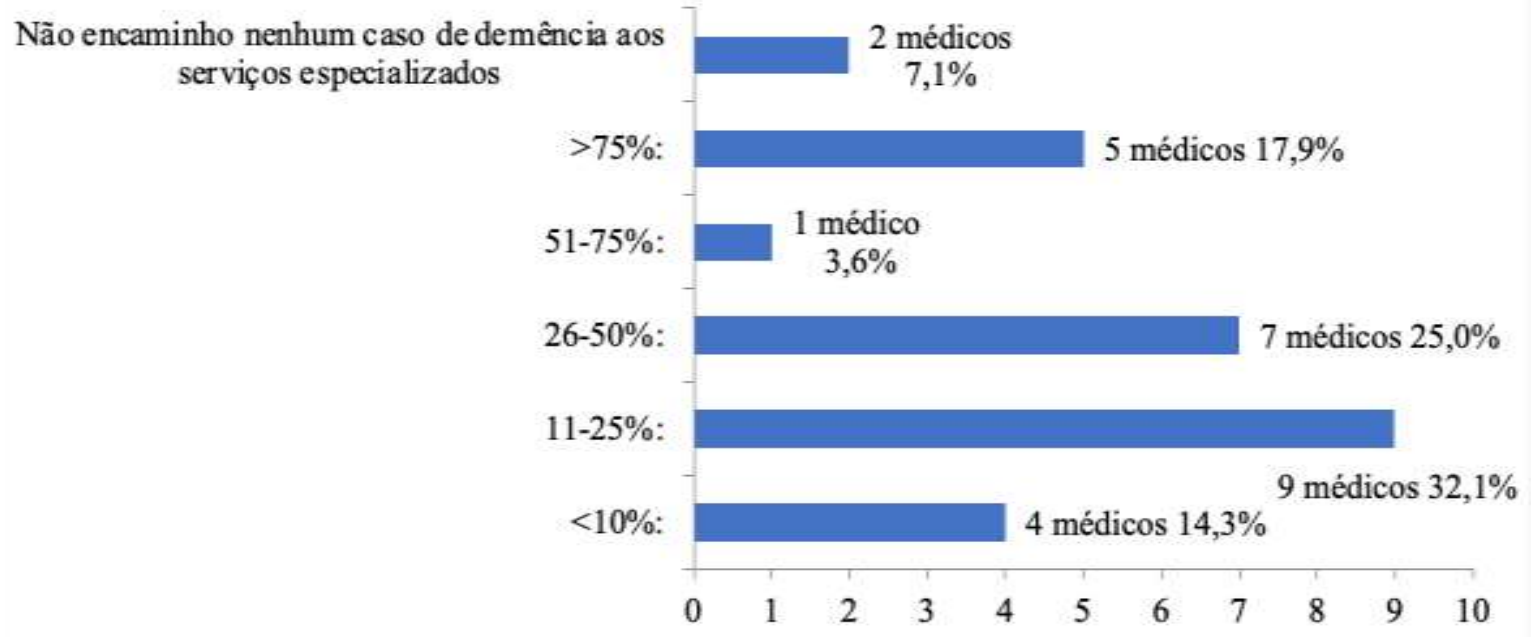

Fonte: Dados de pesquisa.

Partiremos agora para a discussão dos resultados obtidos a partir das respostas do questionário "Instrumento Atenció Sanitària de Les Demències: la visió de L' Atenció Primarià - para médicos - versão brasileira”.

\section{Discussão}

Nesse estudo foi avaliado o perfil médico das equipes de Estratégia de Saúde da Família do município de São Caetano do Sul e, através da aplicação do questionário "Avaliação de conhecimentos e atitudes profissionais no cuidado às demências versão para médicos" pode-se detalhar aspectos sobre o conhecimentos médico sobre demências, características dos atendimentos realizados pelas Unidades Básicas de Saúde em relação a idade dos pacientes atendidos e a presença de comprometimento cognitivo dentre os pacientes. Também foi caracterizado quais as dificuldades apresentadas no diagnóstico, tratamento e manejo de pacientes com demência, bem como a necessidade de realização de exames complementares para diagnóstico diferencial desses quadros, corroborando com dados de estudo realizado anteriormente (Unwin, Loskutova, Knicely \& Wood, 2019).

Através dos resultados, nota-se que apesar de todos os participantes considerarem necessário que haja capacitação específica para atenção no cuidado às demências, somente 17,9\% referem ter realizado atividades com essas características no último ano, o que diverge da proposta do protocolo clínico e diretrizes terapêuticas de demências (Brasil, 2013).

Devido à transição demográfica, os processos relacionados ao envelhecimento, como o comprometimento cognitivo, apresentam uma forte demanda sobre os serviços de saúde em especial a Atenção Básica, o que pode ser evidenciado nesse estudo pelo percentual aproximado de mais de $25 \%$ de pacientes atendidos com mais de 60 anos de idade o que também é evidenciado pelo Estudo de Carga Global de doenças e seu papel na definição de prioridades para a melhoria internacional da saúde pública no próximo século ("Global burden," 2020).

Esse processo ganha uma importância ainda maior no Brasil devido a baixa escolaridade e a maior ocorrência de 
fatores de risco como Hipertensão Arterial Sistêmica, Diabetes Mellitus e Dislipidemia que apresentam relação com aumento da incidência de quadros demenciais conforme relatório realizado pela Comissão Lancet em 2020 que apontou aspectos sobre prevenção, intervenção e cuidado às demências (Livingston et al., 2020).

Deve-se considerar que 78,6\% dos médicos realizam o diagnóstico de demência na fase moderada da evolução do quadro clínico, ou seja, no momento que o paciente apresenta declínio cognitivo importante associado a perda da funcionalidade, na maioria das vezes necessitando de auxílio para as atividades instrumentais da vida diária. Dessa forma, percebe-se que grande parte dos diagnósticos são tardios o que impede a introdução do tratamento no início dos sintomas de declínio cognitivo com melhora da qualidade de vida dos pacientes (Suemoto et al., 2017).

Observou-se que $57,1 \%$ dos médicos referiram que não utilizam habitualmente escalas para avaliação da funcionalidade embora essas sejam importantes para a definição do diagnóstico de síndrome demencial e indicação de tratamento medicamentoso, conforme apontado em estudo (Brucki, 2013).

É possível analisar que $32,7 \%$ dos médicos referiram encaminhar mais que $75 \%$ dos pacientes com síndrome demencial para os serviços de referência o que demonstra a baixa resolutividade da Atenção Básica, diferente do apontado pelo protocolo clínico e diretrizes terapêuticas de demências, o que acarreta aumento de demanda aos serviços secundários e demora nos atendimentos prestados aos pacientes (Brasil, 2013).

Em relação aos encaminhamentos para diagnóstico e/ou controle de pacientes com demência, 89,3\% dos médicos consideram o neurologista de referência como a principal indicação para esses casos. Nota-se que $50 \%$ dos médicos também fazem encaminhamento desses pacientes para os Centros de Referência do Idoso. Nesse estudo, 32,1\% dos médicos referiram encaminhar mais de $75 \%$ dos casos atendidos de quadros demenciais para os serviços de referência para que seja realizado a confirmação do diagnóstico e início do tratamento, o que também difere do proposto pelo protocolo clínico de atendimento às demências (Unwin, Loskutova, Knicely \& Wood, 2019).

A aplicação desse instrumento também permitiu avaliar que 70,4\% dos médicos encontram dificuldades para cuidar de pacientes com demência grave, que usualmente necessitam de serem atendidos por visitas domiciliares por estarem acamados. A dificuldade mais frequentemente apontada foi o tratamento medicamentoso complexo necessário para controle de sintomas neuropsiquiátricos comuns a esse perfil de pacientes, o que poderia ser minimizado através de estratégias de educação continuada conforme apontado nas diretrizes terapêuticas (US Preventive Services Task Force et al., 2020).

\section{Conclusão}

De acordo com os resultados nota-se a dificuldade dos médicos da Atenção Primária de realizar diagnóstico precoce de demência e indicar tratamento adequado o que ocasiona no alto índice de encaminhamentos para o serviço de referência e baixa resolutividade na Atenção Básica.

A atuação médica no que diz respeito aos pacientes com diagnóstico de demência da Atenção Básica permite que seja realizada uma capacitação direcionada sobre essa temática o que favorece o fluxo dos protocolos dos serviços e garante assim melhor assistência para esses pacientes e seus familiares e/ou cuidadores.

É importante ressaltar que o presente estudo apresenta limitações já que avaliou somente o cenário citado, sendo assim fundamental que sejam realizados novos estudos para analisar se o mesmo contexto se repete em outras situações.

Novos estudos são necessários para melhor entendimento sobre a atuação dos médicos na Atenção Primária no cuidado às demências, sendo fundamental a inclusão avaliação da assistência fornecida por toda a equipe multiprofissional a esse perfil de pacientes e também a percepção dos pacientes, familiares e/ou cuidadores sobre o atendimento recebido. 
Research, Society and Development, v. 11, n. 1, e24211124723, 2022

(CC BY 4.0) | ISSN 2525-3409 | DOI: http://dx.doi.org/10.33448/rsd-v11i1.24723

\section{Referências}

American Psychiatric Association. (2014). Manual diagnóstico e estatístico dos transtornos mentais: DSM-5. (5. ed.). Porto Alegre: Artmed.

Bottino, C. M. C. et. al. (2008). Estimate of Dementia Prevalence in a Community Sample from São Paulo, Brazil. Dement Geriatr Cogn Disord, 26(4), 291299. https://doi.org/10.1159/000161053

Brasil. (2012). Instituto Brasileiro de Geografia e Estatística (IBGE). Censo Brasileiro de 2010. https://www.ibge.gov.br/estatisticas/sociais/saude/9662-censodemografico-2010.html?edicao $=9673 \& \mathrm{t}=$ sobre

Brasil. (2013). Ministério da Saúde. Secretaria de Atenção à Saúde. Portaria $N^{o} 1.298$, de 21 de novembro de 2013. https://bvsms.saude.gov.br/bvs/saudelegis/sas/2013/prt1298_21_11_2013.html.

Brucki, S. M. D. (2013). Epidemiology of mild cognitive impairment in Brazil. Dement Neuropsychol, 7(4), 363-366. https://doi.org/10.1590/S198057642013DN74000002

Costa, G. D. (2014). Adaptação transcultural do instrumento Atenció Sanitária de Les Demències: la visió de L 'Atenció Primarià. 2014. Dissertação (Mestrado) Escola de Enfermagem da Universidade de São Paulo, São Paulo. https://doi.org/10.11606/D.7.2014.tde-12122014-110114

Costa, G. D. et al. (2015). Avaliação de conhecimentos e atitudes profissionais no cuidado às demências: adaptação transcultural de um instrumento. Rev Esc Enferm USP, 49(2), 298-308. https://doi.org/10.1590/S0080-623420150000200016

Costa, G. D., Spineli, M. C. D. \& Oliveira, M. A. C. (2019). Professional education on dementias in Primary Health Care: an integrative review. Rev Bras Enferm, 72(4), 1086-1093. https://doi.org/https://doi.org/10.1590/0034-7167-2018-0652

Fornari, L. H. T., Garcia, L. P., Hilbig, A. \& Fernandez, L. L. (2010). As diversas faces da síndrome demencial: como diagnosticar clinicamente? Scient Med, 20(2), 185-193. https://revistaseletronicas.pucrs.br/ojs/index.php/scientiamedica/article/download/5824/5429/0

Freire, M.C.M. \& Pattussi M.P. (2018). Tipos de estudos. In: Estrela, C. Metodologia científica. Ciência, ensino e pesquisa. $3^{\mathrm{a}}$ ed. Porto Alegre: Artes Médicas. p.109-127

Fujihara, S. et al. (2004). Prevalence of presenile dementia in a tertiary outpatient clinic. Arq Neuro-Psiquiatr, 62(3a), 592-595. https://doi.org/10.1590/S0004282X2004000400005

Global burden of 369 diseases and injuries in 204 countries and territories, 1990-2019: a systematic analysis for the Global Burden of Disease Study 2019. (2020). The Lancet, 396(10258), 1204-1222. https://doi.org/10.1016/S0140-6736(20)30925-9

Gusso, G. \& Lopes, J. M. C. (2012). Tratado de medicina de família e comunidade: princípios, formação e prática. v.2. Porto Alegre: Artmed.

Herrera Júnior, E., Caramelli, P. \& Nitrini, R. (1998). Population epidemiologic study of dementia in Catanduva city: state of São Paulo, Brazil. Rev psiquiatr clín, São Paulo, 25(2), 70-73.

Koche, J. C. (2011). Fundamentos de metodologia científica: teoria da ciência e iniciação à pesquisa. Petrópolis: Vozes.

Livingston, G. et al. (2020). Dementia prevention, intervention, and care: 2020 report of the Lancet Commission. The Lancet, 396(10248), 413-446. https://doi.org/10.1016/S0140-6736(20)30367-6

Luchesi, B. M., et al. (2021). Prevalence of risk factors for dementia in middle- and older- aged people registered in Primary Health Care. Dement Neuropsychol, 15(2), 239-247. https://doi.org/10.1590/1980-57642021dn15-020012

Mckhann, G. M. et al. (2011). The diagnosis of dementia due to Alzheimer's disease: recommendations from the National Institute on Aging-Alzheimer's Association workgroups on diagnostic guidelines for Alzheimer's disease. Alzheimers Dement, 7(3), 263-269. https://doi.org/10.1016/j.jalz.2011.03.005

Nitrini, R. \& Bacheschi, L. A. (2005). A Neurologia que todo médico deve saber. São Paulo: Atheneu.

Nitrini, R. et al. (2009). Prevalence of dementia in Latin America: a collaborative study of population-based cohorts. Int Psychogeriatr, 21(4), 622-630. https://doi.org/10.1017/S1041610209009430

Pelegrini, L. N. C. et al. (2019). Diagnosing dementia and cognitive dysfunction in the elderly in primary health care: a systematic review. Dement Neuropsychol, 13(2), 144-153. https://doi.org/10.1590/1980-57642018dn13-020002

Suemoto, C. K. et al. (2017). Neuropathological diagnoses and clinical correlates in older adults in Brazil: a cross-sectional study. PLOS Medicine, 14(3). https://doi.org/10.1371/journal.pmed.1002267

Sunderland, T. et al. (1989). Clock drawing in Alzheimer's disease. A novel measure of dementia severity. J Am Geriatr Soc, 37(8), 725-729. https://doi.org/10.1111/j.1532-5415.1989.tb02233.x

Tuero, G. C. et al. (2011). Percepción, actitudes y necesidades de los profesionales de atención primaria con relación al paciente con demencia. Aten Primaria, 43(11), 585-594. https://doi.org/10.1016/j.aprim.2010.11.015

Unwin, B. K., Loskutova, N., Knicely, P. \& Wood, C. D. (2019). Tools for Better Dementia Care. Fam Pract Manag, 26(1), 11-16. https://pubmed.ncbi.nlm.nih.gov/30645088/

US Preventive Services Task Force et al. (2020). Screening for Cognitive Impairment in Older Adults: US Preventive Services Task Force Recommendation Statement. JAMA, 323(8), 757-763. https://doi.org/10.1001/jama.2020.0435

Vale, F. A. C. \& Miranda, S. J. C. (2002). Clinical and demographic features of patients with dementia attended in a tertiary outpatient clinic. Arq NeuroPsiquiatr, 60(3a) https://doi.org/10.1590/S0004-282X200200040000 